\title{
Ghost Sector in Minimal Linear Covariant Gauge
}

\section{Attilio Cucchieri ${ }^{* a}{ }^{a}$ David Dudal, ${ }^{b c}$ Tereza Mendes, ${ }^{a}$ Orlando Oliveira,${ }^{d}$ Martin Roelfs ${ }^{b}$ and Paulo J. Silva ${ }^{d}$}

${ }^{a}$ Instituto de Física de São Carlos, Universidade de São Paulo, C.P. 369, 13560-970 São Carlos, SP, Brazil

${ }^{b}$ KU Leuven Kulak, Department of Physics, Etienne Sabbelaan 53 bus 7657, 8500 Kortrijk, Belgium

${ }^{c}$ Ghent University, Department of Physics and Astronomy, Krijgslaan 281-S9, 9000 Gent, Belgium

${ }^{d}$ CFisUC, Departamento de Física, Universidade de Coimbra, 3004-516 Coimbra, Portugal E-mail: attiliodifsc.usp.br, david.dudaldkuleuven.be, mendeseifsc.usp.br prlandodfis.uc.pt martin.roelfsdkuleuven.be, psilvaduc.pt

We discuss possible definitions of the Faddeev-Popov matrix for the minimal linear covariant gauge on the lattice and present preliminary results for the ghost propagator.

XIII Quark Confinement and the Hadron Spectrum - Confinement2018

31 July - 6 August 2018

Maynooth University, Ireland

\footnotetext{
* Speaker.
} 


\section{Why Study the Linear Covariant Gauge on the Lattice?}

A big effort has been made in the last decades in order to understand the infrared properties of Yang-Mills theories from the study of their Green's functions, notably the gluon and the ghost propagators (see, e.g., [1] and references therein). To this end, several gauge-fixing conditions have been considered: Landau gauge, Coulomb gauge, $\lambda$-gauge, maximal Abelian gauge, etc. In particular, in Landau gauge, the so-called Gribov-Zwanziger (GZ) confinement scenario has captivated a lot of attention [2]. A natural generalization of Landau gauge is the linear covariant gauge (LCG). Recently, a possible way of extending the GZ approach (in the continuum) to LCG has been proposed [3]. The resulting theory is characterized by an exact nilpotent nonperturbative BRST symmetry $\llbracket$.

For the moment, numerical simulations have focused on the implementation of the LCG on the lattice [5, 6, 0, 8] and on the study of the gluon propagator [7, 9, 10]. On the other hand, it is still an open problem how to define the Faddeev-Popov (FP) matrix for LCG on the lattice. This is the goal of our study. Preliminary results have been presented in [11, 12]. Some early results were also presented in [13].

\section{Minimal Linear Covariant Gauge}

In order to impose the LCG on the lattice, one should first recall that the lattice Landau gauge, which is a special case of the LCG, is obtained by minimizing the functional (see, e.g., [14])

$$
\mathscr{E}_{L G}\left[U_{\mu}, g\right] \equiv-\Re \operatorname{Tr} \sum_{x} \sum_{\mu=1}^{d} U_{\mu}^{g}(x) \equiv-\Re \operatorname{Tr} \sum_{x} \sum_{\mu=1}^{d} g(x) U_{\mu}(x) g^{\dagger}\left(x+e_{\mu}\right) .
$$

One can interpret the Landau-gauge functional as a spin-glass Hamiltonian for the "spin variables" $g(x)$ with a "random interaction" given by the link variables $U_{\mu}(x)$. This suggests the existence of several local minima for $\mathscr{E}_{L G}\left[U_{\mu}, g\right]$. The set of these local minima, known as Gribov copies [2, 15, 16], defines the first Gribov region $\Omega$. From the second variation of the minimizing functional, we define the Landau FP operator [16]

$$
\begin{aligned}
\mathscr{M}^{b c}(x, y) \equiv \sum_{\mu=1}^{d} & \left\{\Gamma_{\mu}^{b c}(x)\left[\delta_{x, y}-\delta_{x+e_{\mu}, y}\right]+\Gamma_{\mu}^{b c}\left(x-e_{\mu}\right)\left[\delta_{x, y}-\delta_{x-e_{\mu}, y}\right]\right. \\
& \left.-\sum_{e=1}^{N_{c}^{2}-1} f^{b e c}\left[A_{\mu}^{e}\left(x-e_{\mu} / 2\right) \delta_{x-e_{\mu}, y}-A_{\mu}^{e}\left(x+e_{\mu} / 2\right) \delta_{x+e_{\mu}, y}\right]\right\},
\end{aligned}
$$

which is symmetric (under the simultaneous exchanges $b \leftrightarrow c$ and $x \leftrightarrow y$ ) and semi-positive definite. In the above equation we used the definition

$$
\Gamma_{\mu}^{b c}(\vec{x}) \equiv \operatorname{Tr}\left[\frac{\lambda^{b} \lambda^{c}+\lambda^{c} \lambda^{b}}{4} \frac{U_{\mu}(\vec{x})+U_{\mu}^{\dagger}(\vec{x})}{2}\right]
$$

where $\left\{U_{\mu}(\vec{x})\right\}$ is the gauge-fixed configuration and the matrices $\lambda^{b}$ are the $N_{c}^{2}-1$ traceless Hermitian generators of the gauge group $\mathrm{SU}\left(N_{c}\right)$, normalized such that $\operatorname{Tr}\left(\lambda^{b} \lambda^{c}\right)=2 \delta^{b c}$. Note that for 
the usual generators $\tilde{\lambda}_{b}$ with normalization $\operatorname{Tr}\left(\tilde{\lambda}_{b} \tilde{\lambda}_{c}\right)=\delta_{b c} / 2$, we have

$$
\tilde{\lambda}_{b}=\frac{\lambda_{b}}{2} .
$$

We also defined the lattice gauge field through the relations

$$
\begin{aligned}
& A_{\mu}\left(\vec{x}+\vec{e}_{\mu} / 2\right) \equiv \frac{1}{2 i}\left[U_{\mu}(\vec{x})-U_{\mu}(\vec{x})^{\dagger}\right]-\mathbf{1} \frac{\operatorname{Tr}}{2 i N_{c}}\left[U_{\mu}(\vec{x})-U_{\mu}(\vec{x})^{\dagger}\right], \\
& A_{\mu}^{b}\left(\vec{x}+\vec{e}_{\mu} / 2\right)=\frac{1}{2} \operatorname{Tr}\left[A_{\mu}\left(\vec{x}+\vec{e}_{\mu} / 2\right) \lambda_{b}\right],
\end{aligned}
$$

where $\mathbb{\perp}$ is the identity matrix.

It is important to note that one can write the Landau FP operator (2.2) as [11]

$$
\mathscr{M}=\frac{1}{2}\left(\mathscr{M}_{+}+\mathscr{M}_{-}\right)
$$

with

$$
\left(\mathscr{M}_{+} \gamma\right)^{b}(\vec{x}) \equiv-\sum_{\mu=1}^{d}\left[\left(D_{\mu} \gamma\right)^{b}(\vec{x})-\left(D_{\mu} \gamma\right)^{b}\left(\vec{x}-\vec{e}_{\mu}\right)\right] \equiv-\sum_{\mu=1}^{d}\left[\nabla_{\mu}^{(-)}\left(D_{\mu} \gamma\right)\right]^{b}(\vec{x})
$$

and

$$
\left(\mathscr{M}_{-} \gamma\right)^{b}(\vec{x}) \equiv \sum_{\mu=1}^{d}\left\{D_{\mu}^{T}\left[\gamma^{b}\left(\vec{x}+\vec{e}_{\mu}\right)-\gamma^{b}(\vec{x})\right]\right\} \equiv \sum_{\mu=1}^{d}\left[D_{\mu}^{T}\left(\nabla_{\mu}^{(+)} \gamma\right)\right]^{b}(\vec{x}) .
$$

Here,

$$
D_{\mu}^{b c}(\vec{x}, \vec{y}) \equiv \Gamma_{\mu}^{b c}(\vec{x})\left[\delta_{\vec{x}+\vec{e}_{\mu}, \vec{y}}-\delta_{\vec{x}, \vec{y}}\right]-\sum_{e=1}^{N_{c}^{2}-1} f^{b e c} A_{\mu}^{e}\left(\vec{x}+\vec{e}_{\mu} / 2\right)\left[\delta_{\vec{x}+\vec{e}_{\mu}, \vec{y}}+\delta_{\vec{x}, \vec{y}}\right]
$$

is the lattice gauge-covariant derivative [16], which implies the transpose lattice gauge-covariant derivative

$$
\begin{aligned}
\left(D_{\mu}^{T}\right)^{b c}(\vec{x}, \vec{y}) \equiv \Gamma_{\mu}^{b c}\left(\vec{x}-\vec{e}_{\mu}\right) \delta_{\vec{x}-\vec{e}_{\mu}, \vec{y}}-\Gamma_{\mu}^{b c}(\vec{x}) \delta_{\vec{x}, \vec{y}} & \\
& +\sum_{e=1}^{N_{c}^{2}-1} f^{b e c}\left[A_{\mu}^{e}\left(\vec{x}+\vec{e}_{\mu} / 2\right) \delta_{\vec{x}, \vec{y}}+A_{\mu}^{e}\left(\vec{x}-\vec{e}_{\mu} / 2\right) \delta_{\vec{x}-\vec{e}_{\mu}, \vec{y}}\right]
\end{aligned}
$$

We have also indicated with $\nabla_{\mu}^{(+)}$[respectively $\nabla_{\mu}^{(-)}$] the usual forward (respectively backward) lattice derivative. Since the transpose of the backward lattice derivative $\nabla_{\mu}^{(-)}$is given by $-\nabla_{\mu}^{(+)}$,

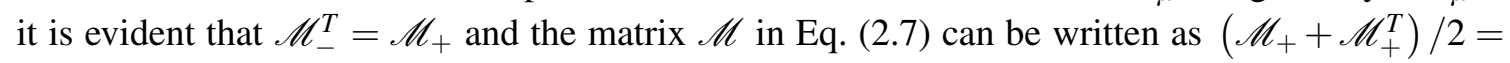
$\left(\mathscr{M}_{-}^{T}+\mathscr{M}_{-}\right) / 2$, which is clearly symmetric (and real).

In turn, the lattice LCG condition can be obtained by minimizing the functional []]

$$
\mathscr{E}_{L C G}\left[U_{\mu}, g, \Lambda\right]=\mathscr{E}_{L G}\left[U_{\mu}, g\right]+\Re \operatorname{Tr} \sum_{x} i g(x) \Lambda(x),
$$


where the functions $\Lambda^{b}(x)$ are real-valued, generated using a Gaussian distribution with width $\xi^{1 / 2}$. By considering a one-parameter subgroup

$$
g(x, \tau)=\exp \left[i \tau \sum_{b=1}^{N_{c}^{2}-1} \gamma^{b}(x) \lambda^{b}\right]
$$

it is easy to check that the stationarity condition implies the lattice LCG condition

$$
\nabla \cdot A^{b}(x) \equiv \sum_{\mu=1}^{d} A_{\mu}^{b}\left(x+e_{\mu} / 2\right)-A_{\mu}^{b}\left(x-e_{\mu} / 2\right)=\Lambda^{b}(x),
$$

which gives, in the formal continuum ${ }^{1}$ limit $a \rightarrow 0$, the gauge condition $a \sum_{\mu} \partial_{\mu} A_{\mu}^{b}(x)=\Lambda^{b}(x)$. It is important to stress that the LCG functional $\mathscr{E}_{L C G}\left[U_{\mu}, g, \Lambda\right]$ is linear in the gauge transformation $\{g(x)\}$. This allows us to naturally extend to the LCG case the gauge-fixing algorithms used in Landau gauge [17]. On the other hand, the standard compact discretization (2.5) for the gauge field $A_{\mu}^{b}\left(x+e_{\mu} / 2\right)$ implies that this field is bounded, so that $\nabla \cdot A^{b}(x)$ is also a bounded quantity. This is in contrast with the $\Lambda^{b}(x)$ functions, which are generated using a Gaussian distribution and, therefore, are unbounded. Thus, one can face convergence problems when a numerical implementation of the LCG is attempted [7, 8, 97.

The continuum gauge field $\hat{A}_{\mu}(\vec{x})$ is usually defined through the relation

$$
U_{\mu}(\vec{x}) \equiv \exp \left[i \operatorname{iag}_{0} \hat{A}_{\mu}\left(\vec{x}+\vec{e}_{\mu} / 2\right)\right]
$$

where $g_{0}$ is the bare coupling constant. Then, Eq. (2.14) yields $a^{2} g_{0} \sum_{\mu=1}^{d}\left[\partial_{\mu} \hat{A}_{\mu}^{b}(\vec{x})+\mathscr{O}\left(a^{2}\right)\right]=$ $\Lambda^{b}(\vec{x})$ in the formal continuum limit $a \rightarrow 0$. On the other hand, the usual gauge field in the continuum limit —i.e. when the generators $\tilde{\lambda}_{b}$ are considered [see Eq. (2.4)] —is given by

$$
2 \hat{A}_{\mu}^{b}(\vec{x}) \approx 2 A_{\mu}^{b}(\vec{x}) /\left(a g_{0}\right)
$$

Thus, with our notation, the continuum functions $\hat{\Lambda}^{b}(\vec{x})$ satisfy the relation $2 \sum_{\mu=1}^{d} \partial_{\mu} \hat{A}_{\mu}^{b}(\vec{x})=\hat{\Lambda}^{b}(\vec{x})$ and we obtain

$$
a^{2} g_{0} \hat{\Lambda}^{b}(\vec{x}) \approx 2 \Lambda^{b}(\vec{x}) .
$$

Then, in the limit $a \rightarrow 0$, it is easy to show [11] that the expression

$$
\frac{1}{2 \xi} \sum_{x} \sum_{b=1}^{N_{c}^{2}-1}\left[\Lambda^{b}(x)\right]^{2} \equiv \frac{\beta}{N_{c} \hat{\xi}} \sum_{x} \sum_{b=1}^{N_{c}^{2}-1} \frac{\left[2 \Lambda^{b}(x)\right]^{2}}{4}
$$

becomes

$$
\frac{1}{2 \hat{\xi}} \int d^{d} x \sum_{b=1}^{N_{c}^{2}-1}\left[\hat{\Lambda}^{b}(x)\right]^{2} .
$$

Here, $\beta=2 N_{c} /\left(a^{4-d} g_{0}^{2}\right)$ in the lattice parameter entering the Wilson action for the $\mathrm{SU}\left(N_{c}\right)$ case, in a generic $d$-dimensional space. Thus, the continuum and lattice widths, $\hat{\xi}^{1 / 2}$ and $\xi^{1 / 2}$, of the corresponding Gaussian distributions are related through the expression $\xi \equiv \hat{\xi} N_{c} /(2 \beta)$. This gives

\footnotetext{
${ }^{1}$ As usual, we indicate with $a$ the lattice spacing.
} 
$\xi<\hat{\xi}$ when $N_{c}<2 \beta$. This inequality is satisfied for $N_{c}=2,3$ and for typical values of $\beta$ in the scaling region.

A few numerical studies of the gluon propagator have been carried out, using the above lattice formulation for LCG [7, 90. In particular, it has been checked, for the SU(2) and SU(3) gauge groups, that the longitudinal propagator $D_{l}\left(p^{2}\right)$ satisfies the relation $p^{2} D_{l}\left(p^{2}\right)=\xi$, as predicted by perturbation theory. At the same time, the transverse gluon propagator $D_{t}\left(p^{2}\right)$ has shown a clear dependence on the gauge parameter $\xi$, i.e. $D_{t}(0)$ decreases as $\xi$ increases. Finally, $D_{t}(0)$ decreases if the lattice volume $V$ increases [ [7], as in Landau gauge. These results are in agreement with the numerical data obtained in Refs. [6, 10], using a different formulation for the lattice LCG, and with several analytic predictions [3, 18, 19].

\section{The Ghost Sector}

In order to define the ghost sector in lattice minimal LCG, one should first recall that, in the continuum, there are in principle three different possible setups for the LCG (see, e.g., Appendix A in Ref. [20]):

1) complex ghost fields $\bar{c}=c^{\dagger}$, giving the the FP matrix $-\partial \cdot D^{b c}$ and a non-Hermitian Lagrangian density;

2) complex ghost fields $\bar{c}=c^{\dagger}$ and a symmetric FP matrix $-\left(\partial \cdot D^{b c}+D^{b c} \cdot \partial\right) / 2$, with a quartic ghost self-interaction term in the Lagrangian density;

3) real independent ghost/anti-ghost fields $u, i v$ and the effective Hermitian FP matrix

$$
\frac{i}{2}\left(\begin{array}{cc}
0 & -\partial \cdot D^{b c} \\
D^{b c} \cdot \partial & 0
\end{array}\right) \equiv i M
$$

On the lattice, if one follows the same procedure used in Landau gauge -i.e. if one evaluates the second variation of the functional $\mathscr{E}_{L C G}\left[U_{\mu}, g, \Lambda\right]$, defined in Eq. 2.12), using the one-parameter subgroup (2.13) - it is immediate to see [11, 12, 13] that the term $\operatorname{ig}(x) \Lambda(x)$ does not contribute to the FP matrix. Thus, one is left with the second variation of the (Landau-gauge) term $\mathscr{E}_{L G}\left[U_{\mu}, g\right]$, yielding the usual symmetric Landau FP matrix $\mathscr{M}$, defined in Eq. (2.2). As we have seen in the previous section, this FP matrix can also be written as

$$
\mathscr{M}=-\sum_{\mu=1}^{d} \frac{1}{2}\left[\nabla_{\mu}^{(-)} D_{\mu}+D_{\mu}^{T}\left(\nabla_{\mu}^{(-)}\right)^{T}\right],
$$

which corresponds to the symmetric FP matrix of case 2) above. On the other hand, it is not clear how a quartic ghost self-interaction term could be obtained on the lattice using the approach considered here. Let us stress that the above matrix $\mathscr{M}$ has real non-negative eigenvalues and real eigenvectors, since it is real and symmetric (and, therefore, it is Hermitian).

A possible lattice discretization of the FP matrix $-\partial \cdot D^{b c}$ of case 1 ) is given by the matrix in Eq. (2.8), which can be written as

$$
\mathscr{M}_{+}^{b c}(x, y) \equiv \mathscr{M}^{b c}(x, y)+\sum_{e=1}^{N_{c}^{2}-1} f^{b e c} \Lambda^{e}(x) \delta_{x, y},
$$


where we used the gauge condition (2.14) and $f^{b c e}$ are the (real) structure constants of the $\mathrm{SU}\left(N_{c}\right)$ gauge group, defined through the commutation relations $\left[\lambda^{b}, \lambda^{c}\right] \equiv 2 i \sum_{e=1}^{N_{c}^{2}-1} f^{b c e} \lambda^{e}$. On the other hand, the extra term in Eq. (3.3) is skew-symmetric under the simultaneous exchanges $b \leftrightarrow c$ and $x \leftrightarrow y$, and it cannot be obtained from a second variation, i.e. it should be added by hand! It is important to note that the matrix $\mathscr{M}_{+}$has complex-conjugate eigenvalues (and eigenvectors) with a non-negative real part. See Ref. [11, 12] for more details about this setup.

Finally, when considering the continuum case 3), one should note that the effective FP matrix $M$, defined in Eq. (3.1), is also real and skew-symmetric and, therefore, it cannot be obtained directly from a second variation of any minimizing functional. On the other hand, since $\mathscr{M}^{b c}(x, y)$ is real and symmetric, if one extends to the complex case the bilinear form

$$
\sum x, y \sum_{b, c=1}^{N_{c}^{2}-1} \gamma_{1}^{b}(x) \mathscr{M}^{b c}(x, y) \gamma_{2}^{c}(y)
$$

i.e. if one considers $\gamma_{1}^{b}(x), \gamma_{2}^{b}(x) \in \mathbb{C}$, then the corresponding sesquilinear form

$$
\left(\begin{array}{cc}
\mathscr{M} & i \mathscr{M} \\
-i \mathscr{M} & \mathscr{M}
\end{array}\right)
$$

is a positive semi-definite Hermitian form. Moreover, its imaginary part is skew-symmetric and gives us a natural way of obtaining the FP matrix

$$
M=\frac{1}{2}\left(\begin{array}{cc}
0 & \mathscr{M}_{+} \\
-\mathscr{M}_{+}^{T} & 0
\end{array}\right)=\frac{1}{2}\left(\begin{array}{cc}
0 & \mathscr{M}_{+} \\
-\mathscr{M}_{-} & 0
\end{array}\right),
$$

which is a possible discretization of the matrix defined in Eq. (3.1). In this case, since $M$ is skewsymmetric, its eigenvalues are complex-conjugate and purely imaginary, and they are related to the singular-value decomposition of $\mathscr{M}_{+}$, i.e. to the eigenvalues of $\mathscr{M}_{+}^{T} \mathscr{M}_{+}$.

\section{Numerical Simulations: Ghost Propagator}

We have done some preliminary tests, evaluating the ghost propagator using the FP matrix defined in Eq. (3.3). Since the matrix $\mathscr{M}_{+}^{b c}(x, y)$ is real and not symmetric, we cannot use the conjugate gradient algorithm, as in Landau gauge. Thus, the inversion of the above FP matrix has been done using [21] the bi-conjugate gradient stabilized algorithm, in the SU(2) case, and the generalized conjugate residual, in the SU(3) case. In both cases we used a point source [22]. Simulations have been carried out with $\beta=2.4469$ for SU(2) and $\beta=6.0$ for SU(3), both corresponding [23] to a lattice spacing $a \approx 0.1 \mathrm{fm}$. Preliminary results for these two gauge groups have been presented in Ref. [11, 12]. One clearly sees from these data that the ghost propagator in LCG agrees, within error bars, with the ghost propagator in Landau gauge (for the same lattice setup). This is in qualitative agreement with the theoretical predictions of Ref. [24] but in disagreement with the finding of Refs. [18, 25]. 


\section{Conclusions}

The numerical evaluation of the ghost propagator in LCG, using the FP matrix $\mathscr{M}_{+}^{b c}(x, y)$ defined in Eq. (3.3), seems feasible. For the lattice setup considered in the simulations presented in Ref. [11, 12], the results are essentially in agreement with the corresponding data in Landau gauge. Of course, simulations at larger physical volumes and different gauge-fixing parameters $\xi$ should be done before one can conclude that this is indeed the case. At the same time, it would be important to extend these numerical simulations to the cases 2) and 3), discussed in Sec. B. Finally, one should try to understand how the first Gribov region $\Omega$ can be defined in lattice minimal LCG, i.e. if the GZ approach can be extended to the LCG on the lattice.

\section{Acknowledgments}

A.C. and T. M. acknowledge partial support from CNPq. A.C. also acknowledges partial support from FAPESP (grant \# 16/22732-1). The research of D.D. and M.R. is supported by KU Leuven IF project C14/16/067. O.O. and P.J.S. acknowledge the Laboratory for Advanced Computing at University of Coimbra (http://www.uc.pt/lca) for providing access to the HPC computing resource Navigator. P.J.S. acknowledges support by FCT under contracts SFRH/BPD/40998/2007 and SFRH/BPD/109971/2015. The SU(3) simulations were done using the Chroma [26] and PFFT [27] libraries.

\section{References}

[1] J. Greensite, An introduction to the confinement problem, Lect. Notes Phys. 821 (2011) 1.

[2] N. Vandersickel and D. Zwanziger, The Gribov problem and QCD dynamics, Phys. Rept. 520 (2012) 175 .

[3] M. A. L. Capri, A. D. Pereira, R. F. Sobreiro and S. P. Sorella, Non-perturbative treatment of the linear covariant gauges by taking into account the Gribov copies, Eur. Phys. J. C 75 (2015) no.10, 479.

[4] M. A. L. Capri et al., Exact nilpotent nonperturbative BRST symmetry for the Gribov-Zwanziger action in the linear covariant gauge, Phys. Rev. D 92 (2015) no.4, 045039.

[5] L. Giusti, Lattice gauge fixing for generic covariant gauges, Nucl. Phys. B 498 (1997) 331.

[6] L. Giusti, M. L. Paciello, S. Petrarca and B. Taglienti, Lattice gauge fixing for parameter dependent covariant gauges, Phys. Rev. D 63 (2001) 014501.

[7] A. Cucchieri, T. Mendes and E. M. S. Santos, Covariant gauge on the lattice: A New implementation, Phys. Rev. Lett. 103 (2009) 141602; Simulating linear covariant gauges on the lattice: A New approach, PoS QCD-TNT09 (2009) 009; A. Cucchieri, T. Mendes, G. M. Nakamura and E. M. S. Santos, Gluon Propagators in Linear Covariant Gauge, PoS FACESQCD (2010) 026.

[8] A. Cucchieri, T. Mendes, G. M. Nakamura and E. M. S. Santos, Feynman gauge on the lattice: New results and perspectives, AIP Conf. Proc. 1354 (2011) 45.

[9] P. Bicudo et al., Lattice gluon propagator in renormalizable $\xi$ gauges, Phys. Rev. D 92 (2015) no.11, 114514; Gauge fixing and the gluon propagator in renormalizable $\xi$ gauges, PoS LATTICE2015 (2016) 317. 
[10] L. Giusti et al., Results on the gluon propagator in lattice covariant gauges, Nucl. Phys. B Proc. Suppl. 94 (2001) 805; L. Giusti et al., Quark and gluon propagators in covariant gauges, Nucl. Phys. B Proc. Suppl. 106 (2002) 995.

[11] A. Cucchieri et al., Faddeev-Popov Matrix in Linear Covariant Gauge: First Results, arXiv:1809.08224 [hep-lat], to appear in Phys. Rev. D.

[12] A. Cucchieri et al., Lattice Computation of the Ghost Propagator in Linear Covariant Gauges, arXiv:1811.11521 [hep-lat], to appear in PoS LATTICE2018 252.

[13] E. M. S. Santos, QCD na rede: um estudo não-perturbativo no calibre de Feynman, doctoral thesis (in Portuguese), University of São Paulo (2011), http://www.teses.usp.br/teses/disponiveis/76/76131/tde19102011-135900/en.php.

[14] L. Giusti et al., Problems on lattice gauge fixing, Int. J. Mod. Phys. A 16 (2001) 3487.

[15] V. N. Gribov, Quantization of Nonabelian Gauge Theories, Nucl. Phys. B 139 (1978) 1.

[16] D. Zwanziger, Fundamental modular region, Boltzmann factor and area law in lattice gauge theory, Nucl. Phys. B 412 (1994) 657.

[17] A. Cucchieri and T. Mendes, Critical slowing down in SU(2) Landau gauge fixing algorithms, Nucl. Phys. B 471 (1996) 263; Study of critical slowing down in SU(2) Landau gauge fixing, Nucl. Phys. B Proc. Suppl. 53 (1997) 811; Critical slowing down in SU(2) Landau gauge fixing algorithms at beta= infinity, Comput. Phys. Commun. 154 (2003) 1; Gauge Fixing in Lattice Minimal Linear Covariant Gauge, in preparation.

[18] M. Q. Huber, Gluon and ghost propagators in linear covariant gauges, Phys. Rev. D 91 (2015) no.8, 085018; A. C. Aguilar, D. Binosi and J. Papavassiliou, Yang-Mills two-point functions in linear covariant gauges, Phys. Rev. D 91 (2015) no.8, 085014.

[19] F. Siringo and G. Comitini, Gluon propagator in linear covariant R $\xi$ gauges Phys. Rev. D 98 (2018) no.3, 034023 .

[20] R. Alkofer and L. von Smekal, The Infrared behavior of QCD Green's functions: Confinement dynamical symmetry breaking, and hadrons as relativistic bound states, Phys. Rept. 353 (2001) 281.

[21] Y. Saad, Iterative Methods for Sparse Linear Systems, SIAM, Philadelphia (USA), 2003, 2nd edition.

[22] P. Boucaud et al., Asymptotic behavior of the ghost propagator in SU3 lattice gauge theory, Phys. Rev. D 72 (2005) 114503; A. Cucchieri, A. Maas and T. Mendes, Exploratory study of three-point Green's functions in Landau-gauge Yang-Mills theory, Phys. Rev. D 74 (2006) 014503.

[23] A. Cucchieri, T. Mendes, O. Oliveira and P. J. Silva, Just how different are SU(2) and SU(3) Landau propagators in the IR regime?, Phys. Rev. D 76 (2007) 114507.

[24] F. Siringo, Gluon propagator in Feynman gauge by the method of stationary variance, Phys. Rev. D 90 (2014) no.9, 094021.

[25] A. C. Aguilar and J. Papavassiliou, Infrared finite ghost propagator in the Feynman gauge, Phys. Rev. D 77 (2008) 125022; M. A. L. Capri et al., More on the nonperturbative Gribov-Zwanziger quantization of linear covariant gauges, Phys. Rev. D 93 (2016) no.6, 065019.

[26] R. G. Edwards and B. Joo [SciDAC and LHPC and UKQCD Collaborations], The Chroma software system for lattice QCD, Nucl. Phys. B Proc. Suppl. 140 (2005) 832.

[27] M. Pippig, PFFT - An extension of FFTW to massively parallel architectures, SIAM J. Sci. Comput. 35 (2013) C213. 